\title{
Who, what, when, where, why
}

\author{
Striking a balance between the need to allocate credit fairly and the need to be readable can \\ be a challenge for editors and journalists when writing about research papers.
}

Here's a challenge. Write a 200-word article about a research paper that explains the main results of the paper - why the work is interesting or important, how the results were obtained, what they mean for that area of nanotechnology and beyond, and who did the work. Such articles - written by editors, journalists and others - appear every week as Research Highlights on the Nature Nanotechnology website ${ }^{1}$, and also in the print issue (p182). Writing these journalistic articles is more difficult than it might seem at first sight: what is interesting or important to one reader might be of little interest or import to another, for instance, and it may be impossible to say anything meaningful about the significance of the results, other than stating that they are indeed significant, in 200 words (or even fewer in some other Nature journals).

Explaining who did the work, one might think, would be easier, but this is far from black and white. Most papers in nanotechnology have four or more authors, often from two or more institutions, and pressures of space mean that it is simply not possible to mention all of these. This is not a problem on research papers (or press releases from universities) although, as discussed below, different tensions and issues arise in the peer-reviewed literature.

On Nature Nanotechnology our policy for non-peer-reviewed articles such as Research Highlights and News \& Views is to refer to the corresponding author of the paper being highlighted in the article, and to mention as many of the institutions as possible (but not the individual departments, centres and so forth within institutions), subject to the space available and the need for the article to remain readable. If the paper does not have a corresponding author, the first author (or the first two authors if their contributions have been equal) is referred to. However, this policy is not without flaws - it raises the profiles of group leaders who always designate themselves as the corresponding author - and it may well be revisited in the future.

In general there is clearly less scope for naming names in a 200-word Research Highlight than there is in a 900-word News \& Views article. This means that the length of the names of institutions can become a factor: if the named author is at the Ecole Supérieure de Physique et de Chimie Industrielles, for instance, it will be more difficult to mention other institutions than if the named author is at, say, Cornell University. This is why we have started to

\section{Explaining who did the work is far from black and white.}

use recognized short-hand names such as Berkeley, Caltech, CNRS, ETH, MIT, NIMS and so on, and why we no longer mention cities or countries in such articles (unless it is clearly preferable to say something like "... and co-workers in France and Spain.")

Of course, it is tempting fate to write this down - which is why it does not appear in our Guide to Authors - because it is impossible to cover all eventualities and because qualitative factors - such as keeping journalistic articles readable and focused on the science rather than the institutional politics - are also involved. Moreover, the Top Down Bottom Up section is an exception to some of these 'rules' because the articles in this section are always about collaborations between researchers with very different backgrounds, so the fact that scientists from Switzerland and China are working together, or that members of a German physics department are collaborating with a healthcare company (p183) is relevant.

The situation is completely different on original research papers, where the names of all the authors and their detailed affiliations are listed. However, given the increasing need to know who did what on papers for a variety of reasons - such as deciding on tenure, curbing honorary authorship and combating misconduct - it is surprising that authors and journals do not make greater use of author contribution statements. Nature Nanotechnology encourages authors to make such statements - and five of the eight research papers in this issue include one - but they are still the exception rather than the rule in the scientific literature, even though editorials in Nature have been advocating their use since $1999^{2}$ and, more recently, discussing the possibility of making them compulsory ${ }^{3,4}$.

In the physics community the famous Alpher-Bethe-Gamow paper ${ }^{5}$ on the creation of the elements in the early universe is often mentioned in discussions about authorship - Alpher did most of the work but his mentor Gamow included Bethe on the paper as a joke. Although it has generated its fair share of laughs over the years, the joke seriously undermined Alpher's contribution to a very significant paper and, understandably, he remained bitter about it for many years. At the risk of feeding a myth, it can be said in Bethe's defence that he at least understood the paper - something that cannot be said for one HAMS ter Tisha who co-authored a paper $^{6}$ with a very well-known nanoscientist in 2001, although this fact escaped editors and journalists alike at the time.

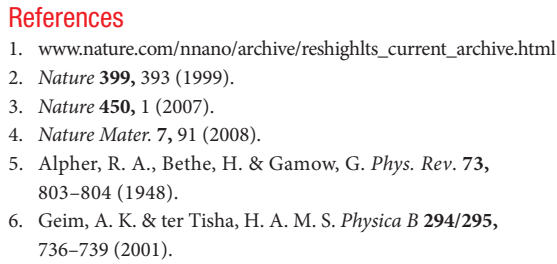

